\title{
Compromises between sonographer and doctor in ultrasound examination assessment and report generation for the sake of better team medicine
}

\author{
Satoshi Yamada ${ }^{1}$ \\ () The Japan Society of Ultrasonics in Medicine 2020
}

I have performed echocardiography at multiple institutions over the years. The need for echocardiography in healthcare is high, and the number of such examinations being performed is increasing at all institutions. While the respective institutions attempt to deal with the increase in the number of such examinations from the standpoint of both personnel and equipment, the efficiency of routine examinations has been improved by division of labor, with a sonographer usually performing ultrasound examinations and a doctor assessing the results. I would like to discuss the routine ultrasound examination assessment and report generation process under such a system. There are probably almost no institutions with a high volume of examinations where a doctor reviews all images recorded by a sonographer before making an assessment. In many cases, it is my guess that a sonographer generates a report and a doctor approves the assessment with almost no confirmation of the images in cases where there are only normal findings or there are no changes from the previous examination. Even in the case of first-time examinations or examinations with new findings, it is my guess that a doctor approves the sonographer's assessment without checking the images or after checking only a few images. I would go so far as to say that the situation today is one where the sonographer generates the entire report, which is supposed to be the responsibility of the doctor, and the doctor then approves the assessment results with almost no changes, without really going into the details of the examination. Of course, there are probably exceptions to this, with some institutions implementing various workarounds, but I will continue this discussion on the assumption that most institutions have found themselves caught up in the above situation. We end up in a situation where

Satoshi Yamada

syamada@tokyo-med.ac.jp

1 Department of Cardiology, Tokyo Medical University Hachioji Medical Center, Tokyo, Japan sonographers want the doctor to make the assessment after confirming as many images as possible from all examinations, while doctors only want to be consulted about problems to be as efficient as possible. What kind of compromise between the two parties can help close this gap?

Sonographer and doctor should communicate well and work in a collaborative manner with a correct understanding of examination methods and the full picture of the pathology or disorder, without limiting the scope of their own work or drawing a boundary between their respective professional duties. A system where busy doctors rubber-stamp reports written by sonographers without having the time to assess the examination results cannot be tolerated. In the case of echocardiography, in particular, there are many acute disorders that need to be considered, and the findings may include those with varying degrees of high urgency. There are cases where a doctor must check the results and make an assessment the same day or immediately after an examination, and cases where a doctor must be present during an examination or be called to an examination and perform it together with the sonographer. A finding that is not expected prior to an examination may be detected incidentally during an ultrasound examination. As such, it is extremely important to have a system in place where a doctor can flexibly check examinations. On the other hand, it is obvious that doctors do not have the time to check all examinations in real time. Therefore, I think it is essential that the sonographer make compromises with the doctor, or with cardiology (in the case of echocardiography). Key points for the sonographer to successfully make compromises are as follows.

1. Some sonographers tend to want doctors to look at all the recorded images from the same perspective as the examiner, but what they should do is first have the doctor get an overall picture via key images, and then consult the doctor about any points that may be of importance in light of the objective of the examination, or specific 
points where the examiner is finding it hard to make a judgment call.

2. In the case of examinations with little in the way of abnormal findings or changes over time, in particular, the sonographer should make use of other approaches like having another sonographer double-check the images, and should only consult the doctor about crucial points.

3. Ultrasonography is inherently a creative task, where the examiner determines the flow of the examination, interpreting the results on the fly and thinking about what to look for next and how to go about it. To do that, the sonographer must have a correct understanding of the clinical objectives required of the examination. The sonographer can contribute to a correct diagnosis and proper treatment by correctly configuring the examination without misjudging the overall flow of care. The emphasis of communication with the doctor should be on the objectives required of the examination as part of the care process.

4. If the above can be achieved, the sonographer will for the most part be able to correctly judge the urgency of findings detected during an examination. If the sonographer can do this, triage of the patient will be possible, and the sonographer can then decide whether the doctor should be called immediately, whether the results should be conveyed the same day, or whether the doctor only needs to check some specific points at a later date. In fact, this kind of decision-making greatly contributes to improving the efficiency of care as a whole.

5. Thus, when we think about what is important in terms of sonographers compromising with doctors, it is apparent that sonographers need to actively insert themselves in care and play a role in pathological assessment and diagnosis of disease. In the area of cardiovascular medicine, in which I work, we encounter many acute disorders, and as such the active participation of the sonographers who perform echocardiography is essential, without which I dare say care as a whole would break down.

Up to this point, I have discussed key points related to compromises between sonographers and doctors in terms of ultrasound examination assessment and report generation. Needless to say, it is important for the doctor to look at the images with their own eyes and understand what the examination entailed [1], but it is also important for the sonographer to get involved in patient care by taking on part of the work that should be done by the doctor. This is essential for improving medical care, and above all, it will make the work of sonographers more rewarding and enjoyable. This is the essence of team medicine. For sonographers who believe that it is the sonographer's job to read findings and the doctor's job to diagnose the patient's condition, all they can do during their limited time with the doctor is present their findings from the images by showing them one by one in order. It is apparent that this is a very old-fashioned notion. The effort and ability to communicate with each other are required to improve team medicine. I also think that public debate to reach a consensus and efforts to come up with rules that focus on the current reality are necessary to tackle this issue.

\section{References}

1. Yamada S. Young doctors trend away from ultrasonography. Jpn J Ultrasonic. 2013;40:461-2.

Publisher's Note Springer Nature remains neutral with regard to jurisdictional claims in published maps and institutional affiliations. 